\title{
Further developments in the CUORICINO experiment
}

S. Pirro , C. Arnaboldi, D.R. Artusa , F.T. Avignone III , M. Balata , I. Bandac , M. Barucci , J.W. Beeman , F. Bellini, C. Brofferio , C. Bucci , S. Capelli , F. Capozzi , L. Carbone, S. Cebrian , M. Clemenza , O. Cremonesi , R.J. Creswick, A. de Waard, M. Dolinski , H.A. Farach , E. Fiorini , G. Frossati, E. Guardincerri , A. Giuliani, P. Gorla , T.D. Gutierrez, E.E. Haller , R. Maruyama , S. Nisi , C. Nones , E.B. Norman , A. Nucciotti , E. Olivieri , M. Pallavicini , V. Palmieri , E. Pasca , M. Pavan , M. Pedretti , G. Pessina , E. Previtali , B. Quiter , L. Risegari , C. Rosenfeld , S. Sangiorgio, M. Sisti , A.R. Smith , L. Torres , G. Ventura

\footnotetext{
Abstract

We report the present results of the CUORICINO experiment, a cryogenic experiment on neutrinoless Double Beta Decay (DBD) of

${ }^{130} \mathrm{Te}$ consisting of an array of $62 \mathrm{TeO}_{2}$ crystals with a total active mass of $40.7 \mathrm{~kg}$. The array is framed inside a dilution refrigerator, heavily shielded against environmental radioactivity and high-energy neutrons, and it is operated at a temperature of $8 \mathrm{mK}$ in the Gran Sasso Underground Laboratory. After several improvements the live time of the experiment is near $75 \%$.
}

\section{Introduction}

The evidence of a neutrino rest mass represents one of the most exciting discovery in the field of particle physics. The discovery of the neutrinoless Double Beta Decay ( $0 v$ -
DBD), however, will provide not only the ultimate answer about the nature (Dirac or Majorana) of the neutrino, but will also allow a sensitivity on the mass down to a few meV. As pointed out very recently by the Members of the APS Multidivisional Neutrino Study [1], Double Beta Decay searches will play the central role in neutrino physics of the next decade. The use of the bolometer technique offers the unique possibility to investigate different DBD nuclei with 
a considerable high energy resolution, needed for future experiments. CUORICINO represents not only the first stage of CUORE [2], but also the most massive DBD Experiment presently running.

\section{Experimental set-up}

The array of CUORICINO consists in a tower with 13 planes containing $62 \mathrm{TeO}_{2}$ crystals operating in the same dilution refrigerator used in our previous experiment [3]. As shown in Fig. 1, the structure is as follows: the upper 10 planes and the lowest one are made of 4 crystals of $5 \times 5 \times 5 \mathrm{~cm}^{3}$, while the 11 th and 12 th ones are made of $93 \times 3 \times 6 \mathrm{~cm}^{3}$ crystals. In these two planes, the central crystal is fully surrounded by the nearby ones. It will be useful to investigate the effect of surface contamination of the crystals and of the copper frame using coincidence and anticoincidence analysis. Four of these small crystals are isotopically enriched (two $75 \%$ of ${ }^{130} \mathrm{Te}$ and two $82 \%$ of ${ }^{128} \mathrm{Te}$ ) in order to search for the $2 v$-DBD decay mode with the method of background subtraction. The temperature sensors are Neutron Transmutation Doped Ge thermistors of $3 \times 3 \times 1 \mathrm{~mm}^{3}$, specifically prepared in order to present similar thermal characteristics, and thermally coupled to each crystal with $9-0.6 \mathrm{~mm}$ diameter epoxy glue spots. A resistor of $\sim 50 \mathrm{k} \Omega$, realized with a heavily doped meander on a $1 \mathrm{~mm}^{3}$ silicon chip, is attached to each absorber and acts as a heater to stabilize the gain of bolometer [4]. The tower is mechanically decoupled from the cryostat in order to avoid vibrations from the cryogenic facility inducing noise on the detectors [5]. The tower is therefore connected, through a $25 \mathrm{~cm}$ copper bar passing through the mixing chamber, to a stainless steel spring fixed at the $50 \mathrm{mK}$ plate

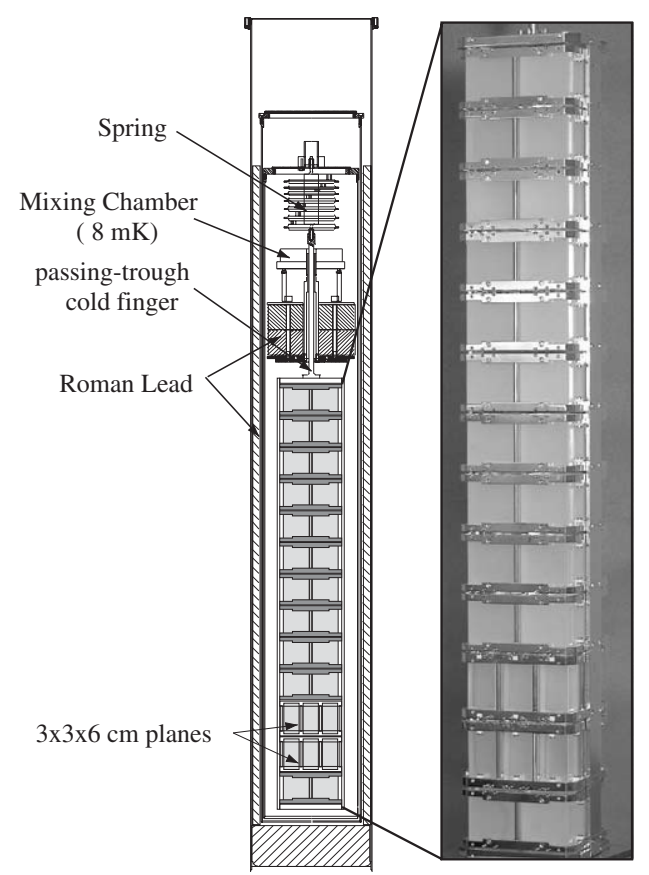

Fig. 1. CUORICINO set-up. (see Fig. 1). The longitudinal oscillation frequency of the system is $\sim 1.8 \mathrm{~Hz}$. The thermal link is ensured by two $40 \times$ $10 \times 0.05 \mathrm{~mm}^{3}$ copper $(99.995 \%)$ foils connected from the $\mathrm{MC}$ to the tower. The heat conductance between MC and holder is $\sim 4.2 \times 10^{-5} \mathrm{~W} / \mathrm{K}$ at $8 \mathrm{mK}$. The temperature of the tower is stabilized through an especially designed feedback device [6].

\section{Physics results}

CUORICINO first measurement started in March 2003. Unfortunately some electrical connections broke during the cooling down procedure, so that only $325 \times 5 \times 5 \mathrm{~cm}^{3}$ and $173 \times 3 \times 6 \mathrm{~cm}^{3}$ crystals could be read. Since the active mass was anyway quite large $\left(\sim 30 \mathrm{~kg}\right.$ of $\left.\mathrm{TeO}_{2}\right)$ and the detector performances were quite good data collection was continued for a few months. The average pulse amplitude obtained with the working detectors is of $150 \mu \mathrm{V} / \mathrm{MeV}$ for the $5 \times 5 \times 5 \mathrm{~cm}^{3}$ crystals and $300 \mu \mathrm{V} / \mathrm{MeV}$ for the $3 \times 3 \times 6 \mathrm{~cm}^{3}$ crystals. The average resolution FWHM in the $0 v$-DBD region is evaluated on the $2615 \mathrm{keV}{ }^{208} \mathrm{Tl}$ line measured during calibration with a ${ }^{232} \mathrm{Th}$ source. It is $7.8 \pm 2.8 \mathrm{keV}$ for the bigger size and of $9.1 \pm 3.1 \mathrm{keV}$ for the small size crystals.

At the end of October 2003 CUORICINO was stopped to undergo substantial operations of maintenance and to recover the lost electrical connections and hence increase the number of working detectors. At the end of April 2004 the second run of CUORICINO started. Unfortunately two of the $5 \times 5 \times 5 \mathrm{~cm}^{3}$ crystal wire connections had broken during the cooling down procedure, and two are discarded due to excess noise. Actually we have 40 big crystals over 44 and all the 18 small crystals working with a $\mathrm{TeO}_{2}$ active mass of about $37 \mathrm{~kg}$. The operating temperature was chosen slightly smaller with respect to the first run, so that the average pulse amplitude is of $215 \mu \mathrm{V} / \mathrm{MeV}$ for the $5 \times 5 \times 5 \mathrm{~cm}^{3}$ crystals and $430 \mu \mathrm{V} / \mathrm{MeV}$ for the $3 \times$ $3 \times 6 \mathrm{~cm}^{3}$ crystals. The average resolution FWHM is $7.5 \pm$ $2.9 \mathrm{keV}$ for the bigger size and of $9.6 \pm 3.5 \mathrm{keV}$ for the small size crystals. In August 2004 we decided to remove the helium re-liquefier that continuously filled up the main bath. This decision was taken due to the continuous problems arising from breakdown in some of its parts. The helium refill is, therefore, made every $42-54 \mathrm{~h}$. Thanks to the holder temperature stabilization, the time needed by the system to recover, after a helium refill, a suitable stability is of the order of 1-2 h. Considering also other maintenances as cold traps refilling/cleaning and some other outline work, the duty cycle of the experiment since August 2004 is $\sim 73 \%$. Discarding the time needed for energy calibration measurement (3 days every 3-4 weeks) the total background live time is $63 \%$. The total background spectra collected up to April 2005, corresponding to a total statistic of $14.7 \mathrm{~kg} / \mathrm{yr}$, is presented in Fig. 2. Apart from the ${ }^{60} \mathrm{Co}$ sum line and the ${ }^{208} \mathrm{Tl}$ line, no other unexpected peak is found near the $2528 \mathrm{keV} 0 v$-DBD region of ${ }^{130} \mathrm{Te}$. 


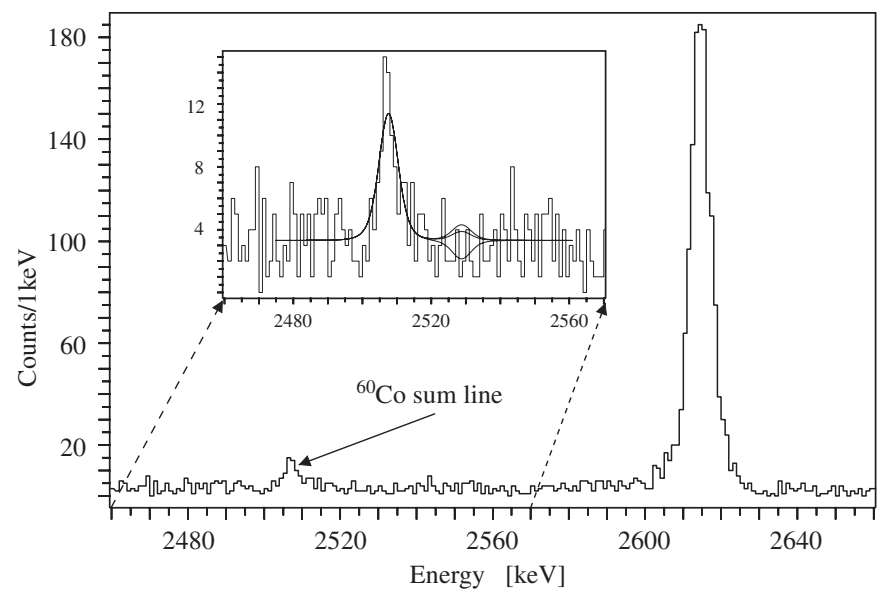

Fig. 2. Background sum spectrum corresponding to a statistic of $14.7 \mathrm{~kg} / \mathrm{yr}$.

The obtained lower limit on the $0 v$-DBD of ${ }^{130} \mathrm{Te}$ is of $1.8 \times 10^{24}$ year ( $90 \%$ C.L.). This limit leads to a constraint on the electron neutrino effective Majorana mass ranging from 0.2 to $1.1 \mathrm{eV}$, depending on the nuclear matrix elements considered in the computation [7].

\section{Conclusions}

Since August 2004 the CUORICINO Experiment is running with a duty cycle of $\sim 70 \%$. Considering the large number of cryogenic detectors involved, this represents a very encouraging result, especially in view of CUORE. The physical result on the limit on the Majorana mass represents the second best limit after Ge detectors (no more running). The projected sensitivity of CUORICINO over 3 years of data acquisition is $6 \times 10^{24}$ year $(68 \%$ C.L.).

\section{References}

[1] S.J. Freedman, B. Kayser (Co-chairs of the organizing committee), arXiv:physics/0411216.

[2] C. Arnaboldi, et al., Nucl. Instr. and Meth. A 518 (2004) 775.

[3] C. Arnaboldi, et al., Phys. Lett. B 557 (2003) 167.

[4] A. Alessandrello, et al., Nucl. Instr. and Meth. A 412 (1998) 454.

[5] S. Pirro, et al., Nucl. Instr. and Meth. A 444 (2000) 331.

[6] C. Arnaboldi, et al., IEEE Trans. Nucl. Sci., 52 (5) (2005) 1630.

[7] C. Arnaboldi, et al., Phys. Rev. Lett. 95 (2005) 142501. 